\title{
EFEITO DO INTEMPERISMO ARTIFICIAL EM QUATRO MADEIRAS TROPICAIS MONITORADO POR ESPECTROSCOPIA DE INFRAVERMELHO (DRIFT)
}

\author{
Tereza Cristina Monteiro Pastore* \\ Laboratório de Produtos Florestais, Serviço Florestal Brasileiro, SCEN Trecho 2, 70818-900 Brasília - DF, Brasil \\ Cynara Caroline Kern de Oliveira e Joel Camargo Rubim
}

Instituto de Química, Universidade de Brasília, CP 04478, 70904-970 Brasília - DF, Brasil

Kelly de Oliveira Santos

Departamento de Química, Universidade Federal de Santa Catarina, 88040-900 Florianópolis - SC, Brasil

Recebido em 26/3/08; aceito em 12/6/08; publicado na web em 10/11/08

\begin{abstract}
EFFECT OF ARTIFICIAL WEATHERING ON TROPICAL WOODS MONITORED BY INFRARED SPECTROSCOPY (DRIFT). Four tropical woods, were investigated to compare their performance and natural resistances to artificial weathering using the diffuse reflectance infrared Fourier transform spectroscopy (DRIFT). The species were garapeira (Apuleia leiocarpa), itaúba (Mezilaurus itauba) and tauari (Couratari sp.), woods traditionally indicated for exterior uses, and marupá (Simarouba amara), that served as reference. The samples were submitted to cycles of UV radiation $(350 \mathrm{~nm})$ and water until 2,000 h. The photodegradation processes of woods surfaces were accomplished monitoring the changes in the intensities associated to lignin $\left(1508 \mathrm{~cm}^{-1}\right)$ and carbonyl group $\left(1736 \mathrm{~cm}^{-1}\right)$ absorptions. The results have shown that lignin was the first component to be photodegradated, resulting in surfaces mainly composed by cellulose, after 2,000 h of UV irradiation. The processes of formation and lixiviation of carbonyl compounds were different for each species. The experimental conditions used in this work were too severe to evaluate the wood density and extractives content influences during the treatment.
\end{abstract}

Keywords: DRIFT; weathering; tropical wood.

\section{INTRODUÇÃO}

Em curto período de tempo, a superfície de uma madeira submetida a intempéries apresenta variação de cor e, posteriormente, torna-se áspera devido à erosão causada pela água da chuva, que remove os polímeros decompostos e deteriora sua microestrutura. ${ }^{1}$ O intemperismo, ação conjunta do sol, da chuva ou umidade e dos ventos, é um processo superficial iniciado pela luz solar que penetra apenas 2,0 $\mathrm{mm}$ abaixo da parte externa da madeira. ${ }^{2}$ A radiação ultravioleta (UV) do espectro solar é o fator considerado mais prejudicial, pois desencadeia reações de decomposição química, levando à formação de radicais livres que atacam os principais constituintes da madeira. ${ }^{3}$ Embora a ação do intemperismo seja superficial, constitui um problema para os usuários de madeira porque afeta tanto a sua aparência estética como o desempenho dos produtos de proteção aplicados sobre ela.

A lignina é o constituinte polimérico da madeira com maior capacidade de absorver energia ultravioleta e visível, pois apresenta uma banda de absorção máxima em $280 \mathrm{~nm}$ que se estende até a região do visível, acima de $400 \mathrm{~nm}$. A absorção da luz leva à formação de radicais livres que reagem com o oxigênio e produzem grupos carbonílicos e carboxílicos cromóforos, ${ }^{2}$ que por sua vez promovem a variação de cor da madeira. Normalmente, primeiro a coloração da madeira sob irradiação sofre escurecimento e, em seguida, clareamento tendendo a acinzentado. A degradação da celulose é mais lenta e é indicada pela perda de peso e redução do grau de polimerização. ${ }^{4}$

Atualmente, os métodos espectroscópicos encontram-se incorporados nos procedimentos de rotina para investigar a composição química da madeira ${ }^{5-8}$ ou de seus constituintes: celulose, ${ }^{9}$ ligninas ${ }^{10-12}$ e glicose e xilose. ${ }^{13}$ Das técnicas de reflexão, a utilizada com maior

*e-mail: tereza.pastore@gmail.com freqüência é a DRIFT, ${ }^{14}$ pois a refletância total atenuada (ATR) necessita que seja estabelecido um contato óptico eficiente entre as superfícies do cristal e da amostra. Muitas vezes, a madeira investigada tem dureza elevada e é resistente ao corte, o que limita o uso da ATR. Mesmo assim, esta técnica vem sendo utilizada para analisar madeiras de clima temperado, ${ }^{15-17}$ que geralmente são menos duras que as de clima tropical.

Vários autores ${ }^{18,19}$ descreveram anomalias e distorções da espectroscopia DRIFT quando os espectros são obtidos diretamente da superfície de um bloco de madeira, como o estreitamento e o deslocamento da banda $\mathrm{OH}$ de 3350 para $3550 \mathrm{~cm}^{-1}$, além do desaparecimento da banda forte localizada em $1030 \mathrm{~cm}^{-1}$. A diluição e a diminuição do tamanho das partículas de madeira em $\mathrm{KBr}$ e o aumento da rugosidade da superfície afetam diretamente a qualidade dos espectros DRIFT. ${ }^{20,21}$ No entanto, essas anomalias não inviabilizaram a utilização do DRIFT, que se mostrou ideal para estudar a decomposição de superfícies causada pelo intemperismo, por manter os componentes in natura, não destruir e nem interferir na superfície tratada, permitindo acompanhar as modificações dos componentes químicos da superfície por comparação das bandas que não são afetadas pelas distorções. . $^{7,21}$

Independente da classificação da árvore, em angiosperma ou gimnosperma, e da espécie estudada, ${ }^{18,19}$ as principais variações da madeira sob intemperismo ocorrem na banda em cerca de $1730 \mathrm{~cm}^{-1}$, atribuída aos grupos carbonílicos das funções aldeído e cetona não conjugados das ligninas e das holoceluloses, e na banda localizada em $1510 \mathrm{~cm}^{-1}$, que é a contribuição das ligações $\mathrm{C}=\mathrm{C}$, incluindo os anéis aromáticos das ligninas e dos compostos extraíveis da madeira. ${ }^{22}$ Algumas propriedades intrínsecas da madeira contribuem com a velocidade de degradação causada pelo intemperismo, tais como a classe a qual pertence (leguminosa ou conífera), ${ }^{18,19}$ a presença de extrativos ${ }^{22}$ sua densidade e anatomia básica. ${ }^{3}$ 
Nesta investigação, o processo de fotodecomposição artificial das espécies florestais tropicais fornecedoras de madeiras - garapeira (Apuleia leiocarpa), itaúba (Mezilaurus itauba) e tauari (Couratari sp.) - por serem tradicionalmente indicadas para uso externo ${ }^{23,24}$ foi acompanhado por DRIFT. O marupá (Simarouba amara) serviu como referência para as outras madeiras, pois contém baixo teor de extrativos e possui coloração branca. $\mathrm{O}$ processo de intemperismo artificial selecionado é mais agressivo para madeiras, pois consistiu em irradiar as amostras com radiação UV $(350 \mathrm{~nm})$ e, em seguida, emergí-las em água sob agitação ao invés de aspergir água sobre suas superfícies, como tradicionalmente é feito pelos equipamentos apropriados. Estudos do comportamento e a extensão da degradação de madeira exposta ao intemperismo artificial dão indicações sobre o seu desempenho quando submetida ao intemperismo natural. Além disto, fornecerão dados de diferentes madeiras que são comparáveis entre si e conduzirão à melhor utilização desse material renovável usado na construção civil em geral.

\section{PARTE EXPERIMENTAL}

\section{Amostragem e preparação dos corpos de prova das madeiras}

Os discos das madeiras, provenientes da Floresta Nacional do Tapajós (PA), encontravam-se armazenados no Laboratório de Produtos Florestais - SFB/Brasília e foram coletados na base do tronco comercial das árvores. Seis amostras medindo cerca de 5,0 x 2,0 x $0,3 \mathrm{~cm}$ foram retiradas do cerne do mesmo disco, em sequiência da casca para a medula, sendo o comprimento no sentido longitudinal, a largura no sentido tangencial e a espessura no sentido radial. As superfícies foram aplainadas e polidas com lixas $n^{\circ} 80$, o que lhes conferiu uma textura grosseira que minimiza a reflexão total.

\section{Instrumentação e processamento dos espectros}

O procedimento de simulação de intemperismo já foi descrito anteriormente ${ }^{25}$ e constituiu basicamente em ciclos de irradiação UV (10 h) seguido de lixiviação em água (2 h) e secagem ao ar livre (3 dias). O tempo de exposição, como expresso nesse trabalho, refere-se a horas de exposição à luz $(0,6,12,20,100,500,1.000$ e 2.000 h), excluindo as horas de lixiviação proporcionais. Após cada período de tratamento foram obtidos os espectros de infravermelho. A vantagem em utilizar o processo de intemperismo artificial é que todas as suas etapas passam a ser reprodutíveis e controláveis.

Os espectros DRIFT das madeiras foram registrados utilizando um interferômetro FTIR Equinox 55 (Brucker, Alemanha), equipado com detector DTGS e acessório de refletância difusa (Harrick Scientific Corporation, USA). Cada espectro representa a média de 64 varreduras com resolução nominal de $4 \mathrm{~cm}^{-1}$. O sinal de referência (background) foi obtido usando um espelho. Para viabilizar comparações quantitativas, os espectros foram normalizados, igualando a altura da banda em ca. $902 \mathrm{~cm}^{-1}$ à unidade. Essa banda é atribuída à celulose, que se mantém invariável após ser irradiada com luz UV. ${ }^{26}$ As intensidades das bandas da carbonila, lignina e celulose foram medidas a partir da linha base, construída conectando-se os pontos mais baixos de cada lado do pico. Uma linha vertical partindo do topo da banda até a linha base forneceu a intensidade da banda.

Os espectros DRIFT foram obtidos como função Kubelka Munk (KM) e processados com o programa GRAMS 5.0 (Galactic). Dessa maneira, assume-se que a concentração dos analitos sobre a superfície da madeira se relaciona diretamente com a intensidade da banda. ${ }^{27}$ Cada espectro representa a média de no mínimo 9 espectros obtidos em pontos diferentes de amostras duplicadas.

\section{RESULTADOS E DISCUSSÃO}

Os espectros DRIFT das madeiras da garapeira, itaúba, marupá e tauari encontram-se ilustrados na Figura 1. São muito semelhantes entre si, como acontece com os polímeros de produtos naturais, porém são de difícil interpretação, pois cada espectro é o resultado de bandas de sobreposição da lignina, da hemicelulose, da celulose e dos extrativos. Apesar da complexidade dos espectros ilustrados, que abrangem toda região do IR médio, somente cerca de 18 bandas podem ser distinguidas para cada madeira estudada, número relativamente pequeno, considerando-se a diversidade e a quantidade de grupos funcionais presentes nos componentes poliméricos.

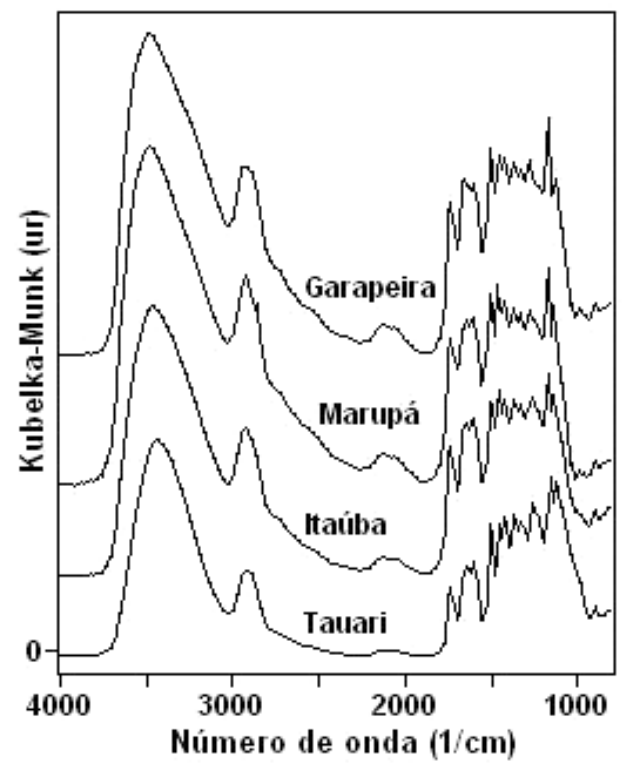

Figura 1. Espectros DRIFT das madeiras estudadas

Todos os espectros mostram uma banda forte de absorção em 3430 $\mathrm{cm}^{-1}[\mathrm{v}(\mathrm{OH})]$ e em cerca de $2900 \mathrm{~cm}^{-1}\left[\mathrm{v}(\mathrm{C}-\mathrm{H})\right.$ e $\left.v\left(\mathrm{CH}_{2}\right)\right]$. Este estiramento $\mathrm{C}-\mathrm{H}$ refere-se ao modo de vibração da ligação $\mathrm{C}-\mathrm{H}$ que possui o carbono com hibridização $\mathrm{sp}^{3}$, como o da celulose. ${ }^{27}$ Cabe destacar que os espectros das madeiras apresentam uma estrutura (ombro) razoavelmente definida em cerca de $3250 \mathrm{~cm}^{-1}$. Este tipo de perfil é característico da presença de compostos carboxílicos e fenólicos, em que ocorre formação de ligação de hidrogênio. Estas estruturas estariam, então, associadas à hemicelulose, aos extrativos e às ligninas.

$\mathrm{Na}$ região imediatamente acima de $1600 \mathrm{~cm}^{-1}$ aparecem três absorções médias bem definidas, em $1739 \mathrm{~cm}^{-1}$ [v(C=O) não conjugada], atribuídas aos grupos carbonílicos das funções aldeído e ceto presentes na lignina e nas xilanas das hemiceluloses, em cerca de $1660 \mathrm{~cm}^{-1}[\mathrm{v}(\mathrm{C}=\mathrm{O})]$, que pode ser causada por outros grupos contendo carbonila na matriz da madeira e também por água absorvida $\left[\delta(\mathrm{HOH})\right.$, deformação angular]. A banda em cerca de $1601 \mathrm{~cm}^{-1}$ $[\mathrm{v}(\mathrm{C}=\mathrm{C})]$ incorpora as contribuições das ligações $\mathrm{C}=\mathrm{C}$ insaturadas, incluindo os anéis aromáticos presentes na lignina.

Embora os espectros das madeiras itaúba, garapeira, marupá e tauari possuam algumas características semelhantes na região da impressão digital, cada madeira tem um padrão infravermelho diferente. Destaca-se nessa região a banda de absorção em cerca de $1510 \mathrm{~cm}^{-1}$, que tem origem nas estruturas dos anéis aromáticos da lignina. ${ }^{9,28-30}$ Estes autores e outros

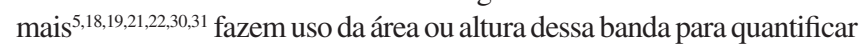
as modificações químicas ocorridas na superfície das madeiras.

As posições das bandas de absorção no infravermelho das quatro madeiras estudadas estão relacionadas na Tabela $1 \mathrm{com}$ as respectivas atribuições, que são gerais e foram feitas por comparação com as 
Tabela 1. Posições das bandas e atribuições dos espectros DRIFT das madeiras estudadas

\begin{tabular}{|c|c|c|c|c|c|c|}
\hline $\begin{array}{l}\text { Garapeira } \\
\left(\mathrm{cm}^{-1}\right)\end{array}$ & $\begin{array}{l}\text { Itaúba } \\
\left(\mathrm{cm}^{-1}\right)\end{array}$ & $\begin{array}{c}\text { Marupá } \\
\left(\mathrm{cm}^{-1}\right)\end{array}$ & $\begin{array}{l}\text { Tauari } \\
\left(\mathrm{cm}^{-1}\right)\end{array}$ & Atribuição $^{\mathrm{a}}$ & Espécie química & Ref. \\
\hline 3466 & 3478 & 3429 & 3479 & $v(\mathrm{O}-\mathrm{H})$ & água; celulose & 5,8 \\
\hline $3307^{b}$ & $3307^{\mathrm{b}}$ & $3304^{\mathrm{b}}$ & $3307^{\mathrm{b}}$ & $v(\mathrm{O}-\mathrm{H})$ & fenóis & \\
\hline 2939 & 2938 & 2931 & 2938 & $v\left(\mathrm{CH}_{2}\right)$ & & $5,8,27$ \\
\hline 2904 & 2909 & 2895 & 2909 & $v\left(\mathrm{CH}_{2}\right)$ & & 8 \\
\hline 1739 & 1737 & 1738 & 1739 & $v(\mathrm{C}=\mathrm{O})$ não conjugada & hemicelulose & $5,8,28$ \\
\hline 1656 & 1651 & 1650 & 1655 & $v(\mathrm{C}=\mathrm{O})$ conjugada; $\delta(\mathrm{HOH})$ & lignina, água absorvida & $5,8,28$ \\
\hline 1602 & 1604 & 1601 & 1602 & $v(\mathrm{C}=\mathrm{C})$ [anel aromático] & lignina & $5,8,28,29$ \\
\hline 1508 & 1512 & 1508 & 1512 & $v(\mathrm{C}=\mathrm{C})$ [anel aromático] & lignina & $5,8,28,29$ \\
\hline 1464 & 1466 & 1460 & 1463 & $\delta(\mathrm{CH})$ & lignina, carboidratos & $5,8,28,29$ \\
\hline 1429 & 1432 & 1425 & 1433 & $\delta(\mathrm{CH}))$ & lignina, carboidratos & 28 \\
\hline 1376 & 1379 & 1372 & 1379 & $\delta(\mathrm{CH})$ & celulose e hemicelulose & 27 \\
\hline 1336 & 1337 & 1328 & 1333 & $\delta(\mathrm{CH}), v\left(\mathrm{C}_{1}-\mathrm{O}\right)$ & celulose, lignina & 5,28 \\
\hline 1317 & - & 1316 & 1318 & $\delta(\mathrm{CH})$ & celulose & 28 \\
\hline 1273 & 1276 & 1270 & 1280 & $v_{\text {as }}(\mathrm{C}-\mathrm{O})$ & ligninas & 5,30 \\
\hline 1238 & 1240 & 1242 & 1239 & $v(\mathrm{C}-\mathrm{O})$ & hemicelulose, lignina & $5,29,30$ \\
\hline 1171 & 1175 & 1163 & 1175 & $\nu_{\text {as }}(\mathrm{C}-\mathrm{O}-\mathrm{C})$ & celulose e hemicelulose & 28,29 \\
\hline 902 & 902 & 900 & 902 & $\gamma(\mathrm{CH}))$ & celulose & 5,28 \\
\hline
\end{tabular}

bandas já descritas na literatura para diferentes espécies folhosas de clima temperado., ${ }^{928-31}$ De modo geral, os valores de números de onda das bandas das quatro madeiras brasileiras descritas na Tabela 1 foram bastante próximos entre si e dos valores observados nos espectros FTIR das madeiras de clima temperado. ${ }^{8,27,28,29,30}$

Durante a fotodegradação da madeira, algumas estruturas químicas são destruídas e outras são formadas, resultando em variações na intensidade das bandas com o aumento do tempo de irradiação UV. Assim como para as leguminosas de clima temperado, as duas principais bandas que sofrem mudanças significativas correspondem ao modo $v(\mathrm{C}=\mathrm{O})\left(1736 \mathrm{~cm}^{-1}\right)$ e ao modo $v(\mathrm{C}=\mathrm{C})\left(1510 \mathrm{~cm}^{-1}\right)$ para as quatro madeiras tropicais estudadas. Outras bandas associadas à lignina em 1600, 1464, 1425, 1330 e $1244 \mathrm{~cm}^{-1}$ também sofrem alterações nas intensidades de absorção, como pode ser observado nos espectros de cada espécie florestal ilustrados na Figura 2.

Em relação à degradação da lignina, as madeiras estudadas apresentaram o mesmo padrão de comportamento: redução contínua da intensidade de absorção com o aumento do tempo de irradiação UV, conforme mostrado na Figura 3. Isto indica que a lignina está sendo consumida durante o processo fotoquímico e, corrobora com essa afirmação, a redução de intensidade de outras duas bandas também associadas à lignina em 1600 e $1464 \mathrm{~cm}^{-1}$, o que pode ser verificado nos espectros da Figura 2. Cabe ressaltar que para facilitar a leitura dos resultados das Figuras 3 e 4, as intensidades de refletância da amostra não irradiada de cada espécie foram igualadas a 100 e os outros valores foram normalizados em relação a ela.

No entanto, diferentemente da degradação da lignina, a formação de compostos contendo carbonila não seguiu um padrão uniforme para as quatro espécies florestais. Para garapeira e tauari a intensidade da absorção em $1736 \mathrm{~cm}^{-1}$ diminui lenta e progressivamente com o aumento do tempo de irradiação. Por outro lado, verificou-se para itaúba e marupá que, durante as primeiras $50 \mathrm{~h}$ de irradiação, ocorre um aumento de cerca de 22 e $16 \%$, respectivamente, seguido por uma redução lenta da intensidade da banda, conforme ilustra a Figura 4. As curvas da garapeira e do tauari apresentam um decréscimo de 1 e $12 \%$, neste mesmo período de irradiação. Vale observar que os compostos carbonilados formados têm origem na fotodegradação da lignina, hemicelulose e extrativos pela radiação UV. A redução da intensidade de absorção associada com a presença da aromaticidade em $1510 \mathrm{~cm}^{-1}$ é mais acentuada após $50 \mathrm{~h}$ de exposição para as quatro madeiras estudadas (Figura 3), desaparecendo totalmente da
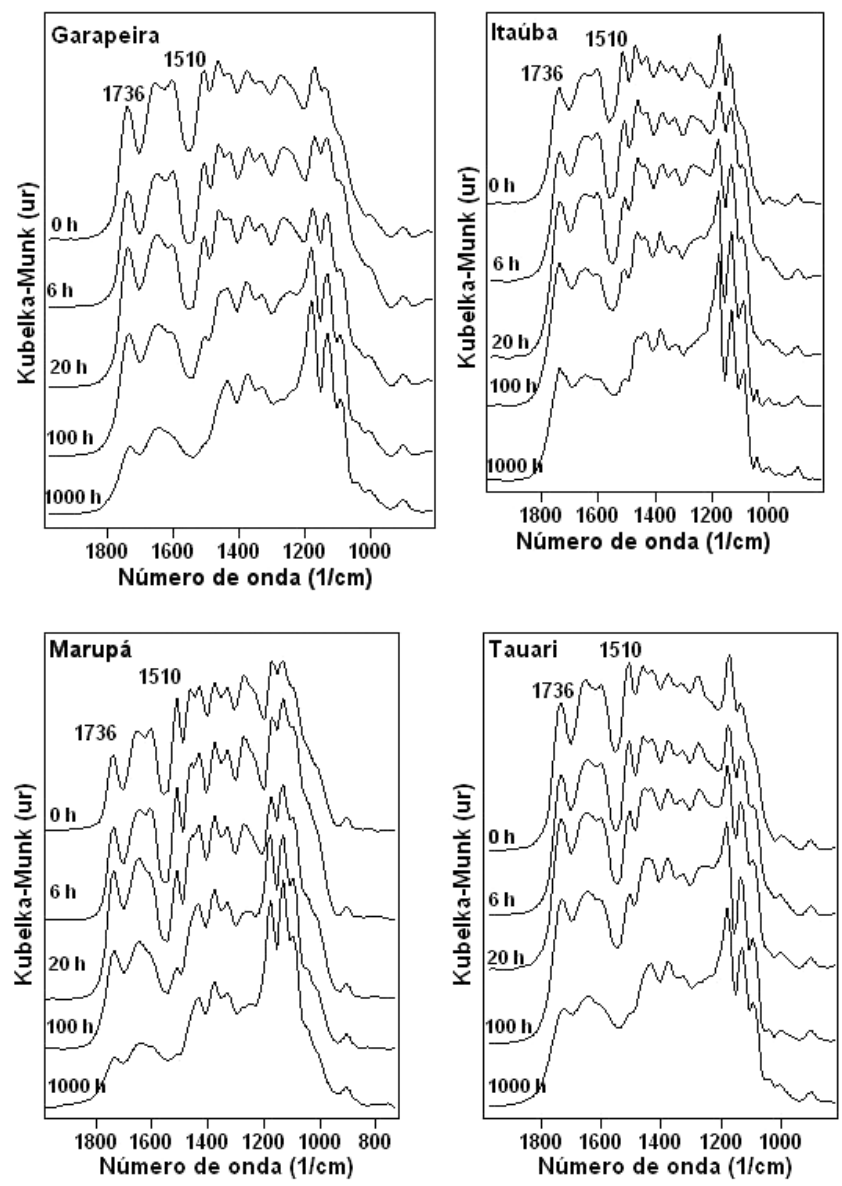

Figura 2. Evolução dos espectros DRIFT das madeiras com o tempo de irradiação UV: (a) $0 \mathrm{~h}$; (b) $6 \mathrm{~h}$; (c) $20 \mathrm{~h}$; (d) $100 \mathrm{he}$ (e) $1000 \mathrm{~h}$ 
superfície do marupá e da garapeira, após 2000 h de exposição. Para a itaúba e tauari restaram somente cerca de 1 e $6 \%$, respectivamente, da concentração inicial da lignina.

Também é aparente pela Figura 3 que, sob as mesmas condições de intemperismo, os efeitos sobre diferentes madeiras ocorrem mais rapidamente para uma espécie que para outra. Por exemplo, o processo de intemperismo para o marupá parece ser mais rápido que para a itaúba, o que pode ser verificado pela intensidade de variação da banda da lignina em $1510 \mathrm{~cm}^{-1}$. Assim, se os valores de intensidade de absorção da lignina após 50 h de irradiação UV forem considerados, há indicativos que a ordem de resistência natural das madeiras estudadas seja a seguinte: tauari>itaúba>garapeira>marupá.

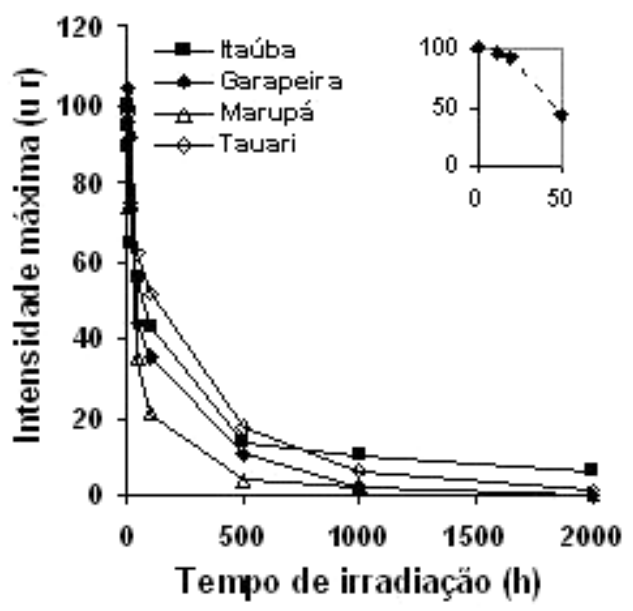

Figura 3. Evolução das variações da intensidade de banda da lignina (1510 $\mathrm{cm}^{-1}$ ) com o tempo de irradiação UV

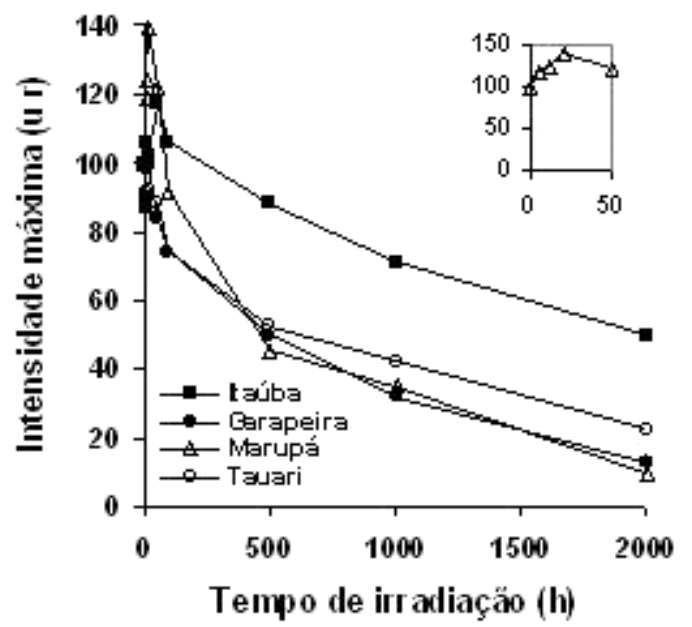

Figura 4. Evolução das variações da intensidade de banda da carbonila $\left(1736 \mathrm{~cm}^{-1}\right)$ com o tempo de irradiação UV

Na Figura 5 estão ilustrados os espectros das quatro madeiras após $2.000 \mathrm{~h}$ de irradiação UV e o espectro da celulose pura microcristalina. Como já verificado anteriormente para outras espécies florestais, ${ }^{20,21}$ observa-se que o padrão espectral geral das superfícies após o tratamento é muito similar para as quatro madeiras que, por sua vez, se assemelham ao da celulose pura. Cabe ressaltar que as superfícies das amostras do marupá ficaram recobertas por uma camada branca, visível a olho nu. Vários pesquisadores afirmam que a celulose é protegida da fotodegradação por coexistir com a lignina que absorve

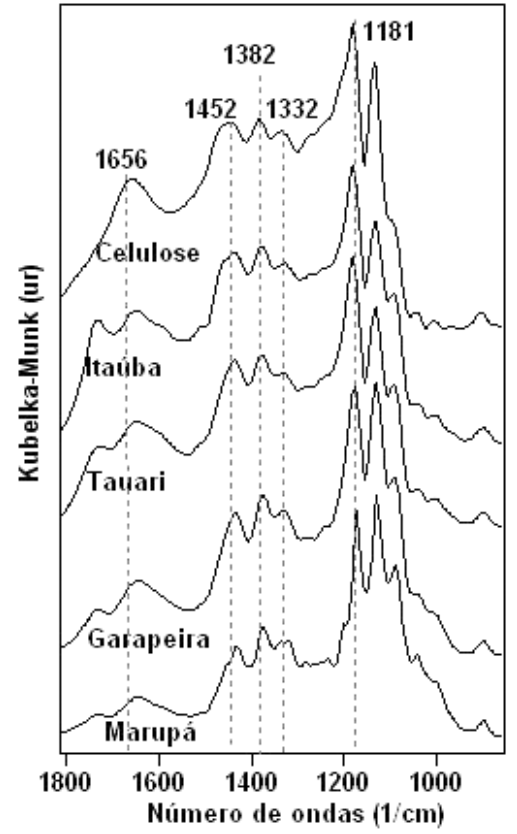

Figura 5. Espectros DRIFT das madeiras após 2.000 h de irradiação UV

preferencialmente a radiação UV e, indiretamente, previne a celulose de ser oxidada por UV, ${ }^{31}$ fato que foi verificado neste trabalho.

Entre outros fatores que contribuem com a velocidade de degradação dos componentes químicos da madeira inclui-se a densidade. ${ }^{20}$ Das madeiras estudadas, o marupá tem baixa densidade básica $\left(0,45 \mathrm{~g} / \mathrm{cm}^{3}\right)$, o tauari $\left(0,56 \mathrm{~g} / \mathrm{cm}^{3}\right)$ e a itaúba $\left(0,72 \mathrm{~g} / \mathrm{cm}^{3}\right)$ apresentam densidade média e a garapeira é pesada $\left(0,86 \mathrm{~g} / \mathrm{cm}^{3}\right) .{ }^{32}$ Apesar de todas essas espécies serem classificadas como madeiras folhosas (hardwoods), o marupá teve um comportamento muito similar ao de uma madeira conífera (softwood),,$^{18}$ tanto na extensão de compostos carbonílicos formados como no desaparecimento dos compostos com aromaticidade. Este fato pode estar relacionado com a baixa densidade do marupá.

Apesar das quatro madeiras poderem ser agrupadas quanto ao teor de extrativos, ${ }^{33}$ o tauari $(1,4 \%)$ e marupá $(2,1 \%)$ com baixos teores, enquanto a itaúba $(10,0 \%)$ e a garapeira $(9,4 \%)$ têm teores mais elevados, nas condições experimentais utilizadas não foi possível estabelecer uma relação entre a quantidade de extrativos com a resistência natural das madeiras.

\section{CONCLUSÕES}

A espectroscopia DRIFT, por ser uma técnica não destrutiva, mostrou-se uma ferramenta adequada para acompanhar o processo de intemperismo simulado de madeiras, por meio da variação da intensidade das bandas associadas à decomposição da lignina e de formação dos compostos carbonílicos.

O comportamento das quatro espécies estudadas sob intemperismo artificial apresentou algumas diferenças significativas em relação à formação e lixiviação dos compostos carbonilados. Porém, para todas as espécies, a lignina foi o componente degradado mais rapidamente, com tendência à diminuição da banda característica $\left(1510 \mathrm{~cm}^{-1}\right)$, que quase desaparece em períodos longos de tratamento.

Para estabelecer uma relação entre as intensidades de absorção dos grupos químicos e a resistência natural das madeiras é necessário utilizar condições experimentais mais brandas que as usadas neste estudo. Dos resultados encontrados, um indicativo sobre o desempenho das madeiras após $50 \mathrm{~h}$ de tratamento, seguiu a seguinte ordem: tauari>itaúba>garapeira>marupá. Outros aspectos como a densidade 
básica da madeira e o teor de extrativos que influenciam na velocidade de degradação também poderão ser avaliados.

\section{AGRADECIMENTOS}

Ao Laboratório de Materiais e Combustíveis/Instituto de Química/UnB pelo uso do reator UV e do FTIR e ao CNPq pela bolsa PIBIC.

\section{REFERENCIAS}

1. Daniel, T.; Hirsch, M. S.; Mcclelland, K.; Ross, A. S.; Williams, R. S.; J. C. T. Coating Tech. 2004, 1, 42.

2. Hon, D.N.-S. Em Wood and cellulosic chemistry; Hon, D.N.-S.; Shiraishi, N., eds.; Marcel Dekker: New York, 2001.

3. Feist, W. C.; Hon, D. N. -S. Em The chemistry of solid wood; Rowell, R. M., ed.; Am. Chem. Soc.: Washington D.C., 1984.

4. Pandey, K. K.; J. Appl. Polym. Sci. 1999, 71, 1969.

5. Pandey, K. K.; Pitman, A. J.; International Biodeterioration and Biodegradation 2002, 52, 151.

6. Weiland, J. J.; Guyonnet, R.; Holz als Roh- und Werkstoff 2003, 61, 216.

7. Mitsui, K.; Murata, A.; Tolvaj, L.; Holz als Roh- und Werkstoff 2004, $62,164$.

8. Deka, M.; Humar, M.; Rep, G.; Kricej, B.; Sentjurc, M.; Petric, M.; Wood Sci. Technol. 2008, 42, 5 .

9. Michell, A. J.; Higgins, G. H.; Infrared spectroscopy in Australian Forest Products Research, CSIRO Forestry and Forest Products: Melbourne, 2002.

10. Schanninger, M.; Hinterstoisser, B.; Holzforschung 2002, 56, 161.

11. Magalhães, W. L. E.; Pereira, J. C. D.; Bolzon Muniz, G. I.; Klock, U.; Silva, J. R. M. da; Anais do 8 Congresso Florestal Brasileiro, São Paulo, Brasil, 2003.

12. Papp, G.; Preklet, E.; Košiková, B.; Barta, E.; Tolvaj, L.; Bohus, J.; Szatmári, S.; Berkesi, O.; J. Photochem. Photobiol. A 2004, 163, 187.

13. Schultz, T. P.; Templeton, M. C.; Mcginnis, G. D.; Anal. Chem. 1985 $57,2867$.
14. Moore, A. K.; Owen, N. L.; Applied Spectroscopy Reviews 2001, 36, 65.

15. Košiková, B.; Sasinková, V.; Tolvaj, L.; Papp, G.; Szatmári, S.; Nagy, T.; Wood Research 2001, 46, 11.

16. Müller, U.; Rätzsch, M.; Schwanninger, M.; Steiner, M.; Zölbl, H.; J. Photochem. Photobiol. B 2003, 69, 97.

17. Nuopponen, M.; Vuorinen, T.; Jämsä, S.; Viitaniemi, P.; Wood Sci. Technol. 2003, 37, 109.

18. Anderson, A. E.; Pawlak, Z.; Owen, N. L.; Feist, W. C.; Appl. Spectrosc. 1991, 45, 641 .

19. Anderson, A. E.; Pawlak, Z.; Owen, N. L.; Feist, W. C.; Appl. Spectrosc. 1991, 45, 648.

20. Anderson, T.; Weaver, F. W.; Owen, N. L.; J. Mol. Struct. 1991, 249, 257.

21. Tolvaj L.; Mitsui, K.; Appl. Spectrosc. 2004, 58, 1137.

22. Pandey, K. K.; Polym. Degrad. Stab. 2005, 87, 375.

23. Instituto Brasileiro de Desenvolvimento Florestal - IBDF; Potencial madeireiro do Grande Carajás, IBDF/LPF: Brasília, 1983.

24. Souza, M. H. de; Madeiras tropicais brasileiras, IBAMA/LPF: Brasília, 1997.

25. Silva, J. O.; Pastore, T. C. M.; Floresta e Ambiente 2004, 11, 7.

26. Horn, B. A.; Qiu, J.; Owen, N. L.; Feist, W. C.; Appl. Spectrosc. 1994, $48,662$.

27. Pastore, T. C. M.; Tese de Doutorado, Universidade de Brasília, Brasil, 2004.

28. Tolvaj, L.; Faix, O.; Holzforschung 1995, 49, 397.

29. Colom, X.; Carrillo, F.; Nogués, F.; Garriga,; P.; Polym. Degrad. Stab. 2003, 80, 543.

30. Sudiyami, Y.; Tsujiyama, S.; Imamura, Y.; Takahashi, M.; Minato, K.; Kajita, Y.; J. Wood Sci. 1999, 45, 348.

31. Dirkx, O.; Triboulot-Trouy, M. C.; Merlin, A.; Deglise, X.; Ann. Sci. For. 1992, 49, 425.

32. Melo, J. E.; Camargos, J. A.; A madeira e seus usos, SFB/LPF: Brasília, 2007 (trabalho não publicado).

33. Santana, M. A. E.; Okino, E. A. Y.; Holzforschung 2007, 61, 469. 